\title{
Introduction to Big Data-Driven Social Media Management Minitrack
}

\author{
Xiangbin Yan \\ University of Science and \\ Technology Beijing, China \\ xbyan@ustb.edu.cn
}

\author{
Mingxin Gan \\ University of Science and \\ Technology Beijing, China \\ ganmx@ustb.edu.cn
}

\author{
Hua (Jonathan) Ye \\ The University of Waterloo, Canada \\ jonathan.ye@uwaterloo.ca
}

\section{Introduction}

Being a necessity of nowadays' life, big data-driven social media has been offering new and meaningful ways to engage global consumers in social communications, personal activities, business behaviors, and professional affairs that matter to them.

Big data-driven social media also carries and propagates essential information that reflects public opinions and personalized preferences on products, events, service, etc., with a huge impact on both individuals and organizations. On the one hand, organizations may need to adopt big data-based techniques to better understand the mechanisms of brand-related opinions, social influence, commercial behaviors, as well as business crises related to social media. On the other hand, big data-driven methodologies have been designed to meet and discover individuals' personalized purposes (e.g., making friends, commodity collaborations, and knowledge acquisition) in social media, supporting their future activities before they make decisions. This minitrack intends to explore and inspirit fresh multidisciplinary studies that offer insights into big data-driven social media management.

This year, three accepted papers investigated how big data-driven techniques in social media provides unique value to both individuals and organizations, and how to effectively adopt methodologies, e.g. big data analysis, for social media management. Topics are focused on Big data-driven social media analysis under COVID-19, Big data-driven intelligent analysis on online impression and Big data-driven contextual analysis for sentiment identification. The introductions of the papers are as below:

\section{Big data-driven social media analysis under COVID-19}

Title: Understanding the Rise of Twitter-based cyberbullying due to COVID-19 through comprehensive statistical evaluation
COVID-19 pandemic has created a challenging situation for everyone, especially starting a digital evolution due to stay-at-home restriction to stop the spread. This has led to an uprise of digital presence, with many hypotheses leading to a rise of cybersecurity attacks, including cyberbullying. To evaluate the significance of COVID-19 on cyberbullying reports, a paper in the minitrack collected 454, 046 of publicly available tweets from Twitter from January 1st, 2020-June 7th, 2020. The authors performed statistical analyses on the collected sample set to understand the situation from a quantitative perspective. They propose a Bayesian estimation of this count data trends via an autoregressive Poisson model which clearly show the upward trend since mid-March, which is reportedly the time from which the stay-at-home orders were widespread globally. The authors also provide a finegrained lag based analysis of the model and contrast their methods with an alternative semi-Bayesian ARARCH model. Overall, such analysis shows somewhat conclusive evidence of the rise around the same time as COVID.

\section{Big data-driven intelligent analysis on online impression}

Title: ExeAnalyzer: A Deep Generative Adversarial Network for Multimodal Online Impression Analysis and Startup Funding Prediction

With the rise of equity crowdfunding platforms, entrepreneurs' online impressions are of great importance to startups' initial funding success. Guided by the design science research methodology, a paper in the minitrack is to design a novel Generative Adversarial Network, namely ExeAnalyzer, to analyze CEOs' online impressions by using multimodal data collected from social media platforms. More specifically, ExeAnalyzer can detect CEOs' first impressions, personalities, and other 
sociometric attributes. Based on a dataset of 7,806 startups extracted from AngelList, another contribution of the paper is the empirical analysis of the relationship between CEOs' online impressions and startups' funding successes. Empirical analysis shows that CEOs' impression of dominance is negatively related to startups' funding performance, while the social desirability of CEOs is positively associated with startups' funding success. Empirical study also confirms that the impression features extracted by ExeAnalyzer have significant predictive power on startups' funding performance.

\section{Big data-driven contextual analysis for sentiment identification}

Title: Identifying sentiment influences evoked by context factors - results from a data analytics procedure performed on tweets
Context factors have lasting impacts on people's sentiments. Exploring impacts that different contexts have on sentiments can be crucial for managing the increasing number of communications companies nowadays maintain with customers via social media channels. To help companies prevent impacts of negative word of mouth, a paper in the minitrack provides an overview about sentiment-influential contexts for tweets as one kind of social media texts previously discussed within the literature. The authors collected an overall amount of 358.923.210 tweets and performed analysis to uncover the effects of continents, mobile devices' operating systems (OS) and the combination of both on sentiments expressed within tweets. Results show remarkable differences for tweets originating from North America and Apple devices, which turned out to be the tweets with the lowest sentiments compared to other continents and the mobile OS Android. 